\title{
Teor lipídico da dieta, lipídios séricos e peso corporal em ratos exercitados
}

\author{
Dietary fat content, serum lipid levels \\ and body weight of exercised rats
}

Larissa Dantas Pereira FRANCO ${ }^{1}$

Juliana Alvares Duarte Bonini CAMPOS²

Aureluce DEMONTE'

\section{RE S U M O}

\section{Objetivo}

Analisar os efeitos de diferentes níveis de triglicerídeos fornecidos pela dieta sobre os parâmetros séricos triglicerídeos e lipoproteínas de alta densidade e o peso corporal de ratos sedentários e exercitados.

\section{Métodos}

Foram utilizados ratos Wistar, alimentados com dietas controle (7\% peso/peso) e hiperlipídica ( $14 \%$ peso/peso) e subdivididos em sedentários e exercitados, por um período de 8 semanas.

\section{Resultados}

Verificou-se que o peso não diferiu entre os grupos, embora o consumo tenha sido reduzido nos grupos com dieta hiperlipídica. Os níveis de triglicerídeos não foram aumentados com a dieta rica em gordura e o HDL-colesterol se elevou apenas entre os animais exercitados que tiveram dieta normolipídica.

\section{Conclusão}

A intensidade e o tempo de exercício físico influenciam de maneira mais aguda os níveis de HDL-colesterol em comparação aos níveis de triglicérides. Sugere-se que a dieta hiperlipídica deveria sobrepor (14\%peso/peso) de gordura para que houvesse alterações relevantes nos parâmetros lipídicos e supostamente um aumento da performance física.

Termos de indexação: Dieta. Exercício. Gorduras na dieta. Ratos Wistar.

\section{A B S T R A C T}

\section{Objective}

This study analyzed the effects of different amounts of dietary triglycerides on the serum lipids triglycerides and high-density lipoproteins and body weight of inactive and exercised rats.

\footnotetext{
1 Universidade Estadual Paulista Júlio de Mesquita Filho, Faculdade de Ciências Farmacêuticas, Departamento de Alimentos e Nutrição. Rod. Araraquara-Jaú, km 1, 14801-902, Araraquara, SP, Brasil. Correspondência para/Correspondence to: L.D.P. FRANCO. E-mail: <larissadpf@yahoo.com.br>.

${ }^{2}$ Universidade Estadual Paulista Júlio de Mesquita Filho, Faculdade de Odontologia de Araraquara. Araraquara, SP, Brasil.
} 


\section{Methods}

Male Wistar rats were divided into two groups: one fed a control diet (7\% fat by weight) and another fed a high-fat diet (14\% fat by weight). These two groups were further divided into two subgroups: one remained inactive and the other exercised for 8 weeks.

\section{Results}

Body weight did not differ among the groups although less food was consumed by the high-fat diet group. Triglyceride levels did not increase with the high-fat diet and HDL-cholesterol only increased in the normal-fat diet, exercised group.

\section{Conclusion}

Physical exercise intensity and duration had a stronger influence on HDL-cholesterol levels than on triglyceride levels. The diet would probably need a higher fat content to actually affect serum lipid levels and supposedly increase physical performance.

Indexing terms: Diet. Exercise. Dietary fats. Rats Wistar.

\section{N T R O D U Ç Ã O}

O tipo de gordura da dieta influencia funções metabólicas e leva a mudanças no peso e/ou na composição corporal, ainda que não haja ingestão hiperenergética ${ }^{1}$. No entanto, muitas controvérsias são encontradas na literatura científica na relação entre a composição lipídica da dieta e o ganho de peso.

Ellis et al..$^{2}$ verificaram que dietas com grandes quantidades de ácidos graxos saturados (AGS) promovem maior acúmulo de gordura, quando comparadas àquelas ricas em ácidos graxos monoinsaturados e poliinsaturados (polyunsaturated fatty acids - PUFAs). Entretanto, alguns estudos relacionam os PUFAs com maior ganho de peso e indução de obesidade em hamsters e ratos, quando comparados com consumo de $\mathrm{AGS}^{3,4}$

Nos dados de 1 kemoto ${ }^{5}$, roedores que consumiram óleo de soja (com composição principal em ácidos graxos $n-6$ ) ganharam mais peso que roedores alimentados com dieta baseada em óleo de peixe, rico em ácidos graxos n-3.

Estudos recentes sugerem que os ácidos graxos $\mathrm{n}-3$ serviriam como controladores da expressão de genes envolvidos no metabolismo lipídico e na adipogênese por serem mediadores importantes na expressão gênica, atuando via receptores proliferadores e ativadores de peroxissomos celulares ${ }^{6}$.
Os PUFAs têm sido relacionados com efeitos hipotrigliceridêmicos, os quais se devem à redução da atividade de enzimas ligadas à síntese de ácidos graxos, como ácido graxo sintase, glicose-6-fosfato desidrogenase e lipase triacilglicerol ${ }^{7}$ e com o aumento da atividade da enzima carnitina palmitoil transferase, relacionada com a oxidação dos ácidos graxos ${ }^{8}$.

Por outro lado, o aumento crescente da obesidade e suas conseqüências metabólicas têm favorecido a prática de exercício físico, visto como um possível fator interveniente na redução de peso. No entanto, os mecanismos dessa ação são ainda inconclusivos, podendo estar relacionados dentre outros, aos níveis de gordura da dieta, ao tipo de exercício ${ }^{9}$ e ao tempo de exposição.

Os efeitos lipolíticos são observados predominantemente em exercícios aeróbios, que levam a um aumento significativo no número e na atividade das mitocôndrias, favorecendo a oxidação de ácidos graxos ${ }^{10}$.

Os exercícios aeróbios de intensidade leve a moderada, como a natação, facilitam a mobilização das gorduras impedindo o acúmulo de lactato e a conseqüente reesterificação dos ácidos graxos; recrutam menor quantidade de fibras do tipo II, que são glicolíticas; e não prejudicam o fluxo sanguíneo dos adipócitos, estimulando a ação da lipase hormônio sensível'11. Essas funções são válidas e comprovadas para adaptações de exercícios aeróbios. 
O Ciclo de Randle (ciclo glicose-ácido graxo), que pode ocorrer durante o exercício físico quando há o aumento da disponibilidade de ácidos graxos, leva à utilização preferencial destes para a geração de energia (oxidação), diminuindo a degradação de glicogênio e a oxidação de glicose ${ }^{10}$.

Newsholme ${ }^{12}$ cita, entretanto que, quando há reposição dietética de carboidratos ocorre a redução da concentração plasmática de ácidos graxos, ocasionando maior utilização de glicose pelo músculo.

As relações entre ingestão de alimentos e alto teor lipídico e exercício físico mostram-se controversas, portanto, objetivou-se analisar os efeitos da ingestão de uma dieta normal e de uma hiperlipídica sobre os parâmetros séricos (triglicérides; lipoproteína de alta densidade-colesterol - HDL-c) e o peso corporal de ratos sedentários e treinados com natação.

\section{MÉ T O D OS}

Foram utilizados ratos machos da linhagem Wistar, recém-desmamados, obtidos do Biotério Central da Faculdade de Medicina da Universidade Estadual Paulista Júlio de Mesquita Filho (UNESP) Botucatu, com peso médio de 88g. Inicialmente todos os animais foram submetidos a uma adaptação de três dias ao ambiente experimental, recebendo dieta padrão de laboratório. Posteriormente, foram divididos em 4 grupos de 10 ratos (NS: dieta normolipídica sem exercício;
NE: dieta normolipídica com exercício; HS: dieta hiperlipídica sem exercício; HE: dieta hiperlipídica com exercício) com consumo de água e dieta ad libitum. As condições ambientais foram controladas sob temperatura de $22^{\circ} \mathrm{C}$, umidade relativa de $65 \%$ e ciclo de claro e escuro de 12 horas por oito semanas. O protocolo experimental foi aprovado pelo Comitê de Ética da Faculdade de Ciências Farmacêuticas de Araraquara, UNESP (Processo n 23/2005).

Foram utilizados dois tipos de dieta, a controle e a hiperlipídica. A dieta controle foi formulada com base nas normas do American Institute of Nutrition (AIN) ${ }^{13}$. A dieta hiperlipídica foi similar à composição da dieta controle, excetuando a quantidade de gordura utilizada, que foi de $14 \%$. A composição das dietas é mostrada na Tabela 1.

A composição centesimal das dietas foi realizada pelos métodos de Bligh-Dyer ${ }^{14}$ para lipídios e pelo Método de Micro-Kjedahl15 para nitrogênio total e proteínas.

Os ratos foram treinados em tanques de $50 \mathrm{~cm}$ de diâmetro e $1,20 \mathrm{~m}$ de comprimento em sessões de 60 minutos, cinco vezes por semana, segundo sistema de natação adaptado para ratos $^{16}$, com a água aquecida, variando de $28^{\circ}$ a $32^{\circ} \mathrm{C}$. O protocolo, considerado de intensidade moderada, encontrava-se entre $25 \%$ a $65 \%$ do máximo consumo de oxigênio ${ }^{10}$. Antes de iniciar o período de experimento, os animais passaram por adaptação durante uma semana, em que o tempo das sessões e a sobrecarga de trabalho

Tabela 1. Composição das dietas consumidas por ratos Wistar durante o período experimental. Araraquara (SP), 2007.

\begin{tabular}{lcccc}
\hline Ingredientes & Dieta controle $(\mathrm{g} / \mathrm{kg})$ & Valor energético $(\mathrm{kcal} / \mathrm{kg})$ & Dieta hiperlipídica $(\mathrm{g} / \mathrm{kg})$ & Valor energético $(\mathrm{kcal} / \mathrm{kg})$ \\
\hline Amido de milho & 579,480 & 2317,920 & 509,480 & 2037,920 \\
Caseína 86\% de proteína & 200,000 & 800,000 & 200,000 & 800,000 \\
Sacarose & 100,000 & 400,000 & 100,000 & 400,000 \\
Mistura mineral & 35,000 & - & 35,000 & - \\
Mistura vitamínica & 10,000 & - & 10,000 & - \\
L-Cistina & 3,000 & - & 3,000 & - \\
Bitartarato de colina & 2,500 & - & 2,500 & - \\
Butil-hidroxi-tolueno & 0,014 & - & 0,014 & - \\
Óleo de soja & 70,000 & 630,000 & 140,000 & 1260,000 \\
\hline Total & 1000,000 & 4147,920 & 1000,000 & 4497,920 \\
\hline
\end{tabular}


(peso na cauda) foram aumentados gradualmente até atingir o estipulado no protocolo. A sobrecarga de trabalho estipulada foi de $5 \%$ da massa corporal. Os animais sedentários foram mantidos na gaiola durante o exercício dos grupos treinados e a alimentação foi retirada durante as sessões de exercício.

A ingestão das dietas foi verificada diariamente, sendo computada pela subtração das sobras de dieta do dia anterior. O peso dos animais também foi mensurado diariamente por balança semi-analítica Gehaka BG 2000.

Ao final de 8 semanas de natação, os ratos foram deixados em jejum durante a noite (12 horas) e sacrificados por decapitação seguindo os Princípios Éticos na Experimentação Animal adotados pelo Colégio Brasileiro de Experimentação Animal. Os animais sedentários foram mortos no mesmo período do dia que os animais exercitados. O sangue foi coletado, centrifugado a 3 000rpm por 10 minutos e o soro foi armazenado em biofreezer para as análises de triglicérides e HDL-colesterol.

As dosagens de triacilgliceróis e HDL-colesterol foram realizadas pelo método enzimático colorimétrico utilizando kit comercial da Bayer, USA, por sistema automático.

Para verificar a influência dos fatores dieta e exercício físico sobre os valores de peso corporal, triglicérides e HDL-colesterol, realizou-se a análise de variância a um fator (ANOVA). Quando a análise de variância apontou diferença estatisticamente significante procedeu-se à comparação das médias pelo Teste de Tukey. Os testes foram aplicados considerando-se um nível de significância de 5\%.

\section{RESULTADOS}

A média de peso $(\mathrm{kg})$ e a estimativa por intervalo de confiança dos animais encontram-se expostas na Figura 1. Pode-se notar que a média de peso dos animais não diferiu entre os grupos sedentários e exercitados, assim como entre os grupos que receberam dieta normolipídica e hiperlipídica $(p=0,5606)$.

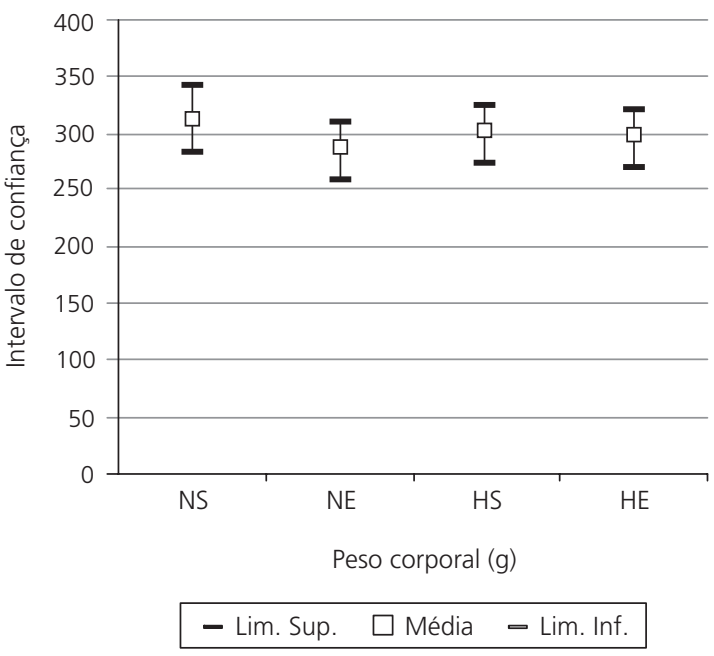

Figura 1. Estimativa do peso corporal (g) por intervalo de confiança de grupos de ratos Wistar. Araraquara (SP), 2008.

Nota: Grupo normolipídico sedentário; NE: grupo normolipídico exercitado; HS: grupo hiperlipídico sedentário; HE: grupo hiperlipídico exercitado; IC: intervalo de confiança 95\%; lim. Sup.: limite superior; lim. Inf.: limite inferior.

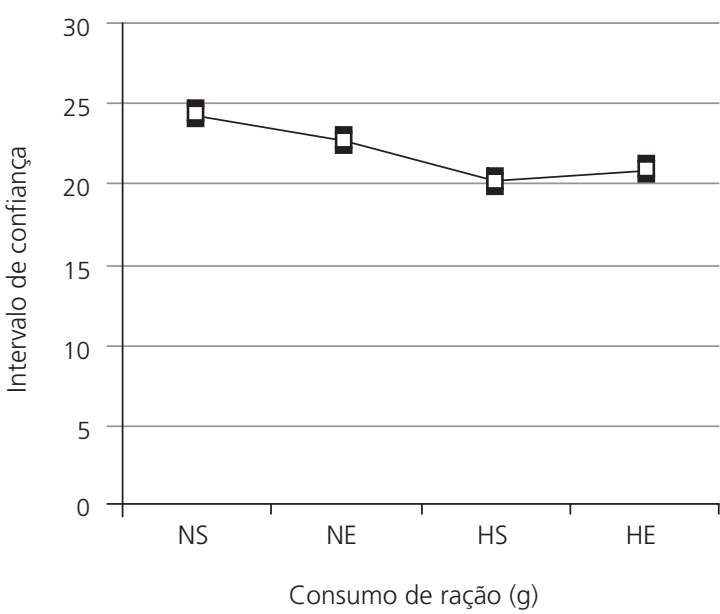

- Lim. Sup. $\quad \square-$ Média $=$ Lim. Inf.

Figura 2. Estimativa do consumo de ração (g) por intervalo de confiança, por grupos de ratos Wistar. Araraquara (SP), 2008.

Nota: Grupo normolipídico sedentário; NE: grupo normolipídico exercitado; HS: grupo hiperlipídico sedentário; HE: grupo hiperlipídico exercitado; IC: intervalo de confiança 95\%; lim. Sup.: limite superior; lim. Inf.: limite inferior. 
Com relação ao consumo alimentar, a estimativa por intervalo de confiança para cada grupo analisado encontra-se na Figura 2. Observa-se diferença estatisticamente significante entre

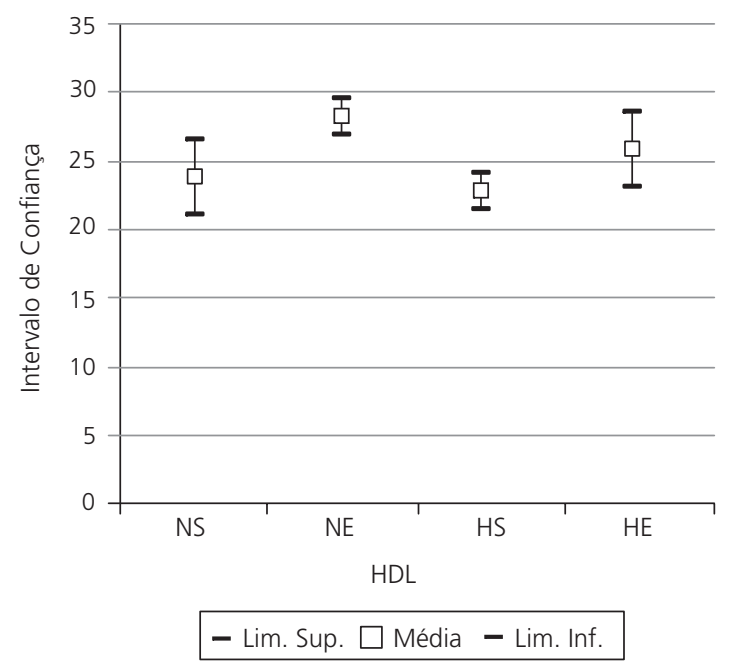

Figura 4. Estimativa do teor de lipoproteína de alta densidade $\mathrm{HDL}$-colesterol $(\mathrm{mg} / \mathrm{dL})$ por intervalo de confiança de grupos de ratos Wistar. Araraquara (SP), 2008.

Nota: Grupo normolipídico sedentário; NE: grupo normolipídico exercitado; HS: grupo hiperlipídico sedentário; HE: grupo hiperlipídico exercitado; IC: intervalo de confiança 95\%; lim. Sup.: limite superior; lim. Inf.: limite inferior.

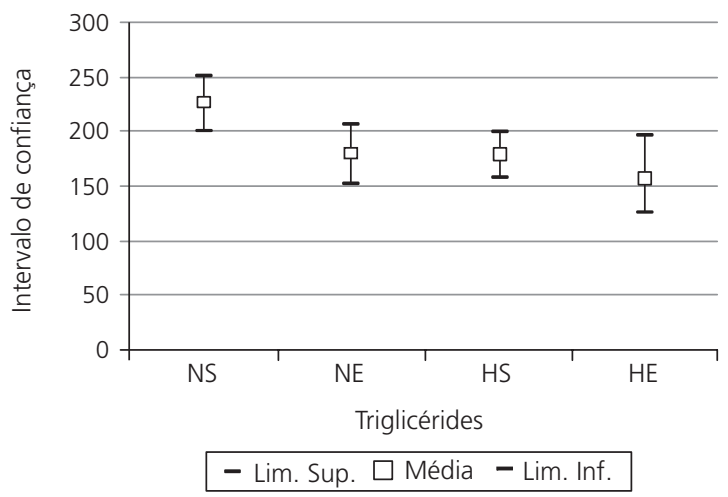

Figura 3. Estimativa do teor de triglicérides (TG) (mg/dL) por intervalo de confiança aferido em grupos de ratos Wistar. Araraquara (SP), 2008.

Nota: Grupo normolipídico sedentário; NE: grupo normolipídico exercitado; HS: grupo hiperlipídico sedentário; HE: grupo hiperlipídico exercitado; IC: intervalo de confiança 95\%; lim. Sup.: limite superior; lim. Inf.: limite inferior. o consumo alimentar nos diferentes grupos $(p<0,0001)$. Nota-se diminuição significante nos grupos HE - dieta hiperlipídica sem exercício, e HS - dieta hiperlipídica sem exercício em relação aos respectivos normolipídicos (NE - dieta normolipídica com exercício e NS - dieta normolipídica sem exercício).

A estimativa por intervalo de confiança do teor de triglicérides $(\mathrm{mg} / \mathrm{dL}$ ) aferido em cada grupo avaliado encontra-se na Figura 3. Observou-se diferença significante no teor de triglicérides entre os grupos $(p=0,008)$, entretanto, testando-se as médias observa-se que a mesma pode ser atribuída aos grupos NS e HE.

Os dados do HDL-colesterol (mg/dL) são indicados na Figura 4. Apesar de não ter ocorrido o efeito hipotrigliceridêmico nos animais exercitados, verificou-se que os níveis de HDL foram significantemente diferentes entre os grupos NS; NE e HS; NE $(p=0,0025)$.

\section{DIS CUSS ÃO}

Frente aos dados de peso corporal, pode-se sugerir que a diferença na quantidade de calorias das duas dietas utilizadas e o tempo do experimento não foram suficientes para induzir a obesidade nos animais, ainda que na dieta do grupo hiperlipídico tenha sido acrescido o dobro da necessidade de gordura. Apesar de acrescentar o dobro de gordura necessária ao animal, o valor energético da dieta hiperlipídica não apresentou grandes diferenças em relação à dieta controle, como se observa na Tabela 1.

Embora os resultados tenham indicado diferenças não-significantes entre os grupos para a evolução ponderal, alguns estudos verificaram efeitos distintos sobre o tamanho das células de gordura e também sobre o número dessas células ${ }^{2}$. Pereira et al. ${ }^{17}$ citam estudos que demonstraram que dietas ricas em ácidos graxos saturados promovem replicação de adipócitos. A indução da hiperplasia de adipócitos com essas dietas levaria a maiores efeitos que a hipertrofia induzida pelas dietas ricas em ácidos graxos insaturados. 
A redução de peso esperada nos animais hiperlipídicos exercitados não ocorreu, resultados também encontrados nos trabalhos de Bell et al. ${ }^{18}$. Os dados de Pellizzon et al. ${ }^{19}$ corroboram o achado, uma vez que ratos alimentados com óleo de soja (rico em ácidos graxos poliinsaturados) e treinados com natação por seis semanas, apresentaram-se mais pesados que ratos com dieta hiperlipídica, podendo, entretanto, ter havido aumento de massa magra e, conseqüentemente, aumento do peso corporal.

O efeito da dieta rica em gordura em auxiliar o desempenho físico e, por conseguinte, levar à redução do peso também não foi confirmado neste experimento. Este resultado pode se dever ao fato de a oxidação de gorduras depender do aporte adequado de oxaloacetato para o Ciclo de Krebs, o qual advém do metabolismo de carboidratos e aminoácidos. Considerando que havia uma baixa disponibilidade de carboidratos, a utilização de aminoácidos para formar intermediários do Ciclo de Krebs aumentou, favorecendo, então, a formação excessiva de amônio $\left(\mathrm{NH}_{3}^{+}\right)$. Esse produto colabora com a fadiga, levando à diminuiç̧ão da oxidação de substratos energéticos.

Conforme Cheng et al. ${ }^{20}$, os efeitos ergogênicos dos lipídios também podem ser dose-dependentes, assim como podem estar relacionados com o tipo de gordura fornecido pela dieta.

Stich et al. ${ }^{21}$ demonstraram ainda que, trabalhos aeróbios intermitentes (com períodos de repouso semelhantes entre uma sessão e outra) foram mais eficientes na mobilização dos ácidos graxos que uma sessão única de esforço físico.

Dessa forma, o que pode ter ocorrido, de acordo com Romijn et al. ${ }^{22}$, é que a lipólise e a conseqüente liberação de ácidos graxos para a circulação é mais elevada durante exercícios de baixa intensidade. No entanto, a hipótese de maior oxidação de triglicerídeos intramusculares durante o exercício, e não daqueles armazenados nos adipócitos, reflete elevada lipólise desses triglicerídeos com o aumento da intensidade do exercício $^{23}$. Portanto, pode-se supor, que para efeito de redução de peso, seria necessário um exercício de intensidade moderada a intensa e um maior tempo de duração.

A diferença estatisticamente significante entre o consumo alimentar nos diferentes grupos sugere aumento da saciedade, dados que se assemelharam aos de Kretschmer et al. ${ }^{24}$. Estudos prévios indicam que dietas ricas em PUFAs têm alta eficiência e reduzem, assim, a ingestão alimentar ${ }^{25}$. Com relação à presença de exercício físico, verificou-se uma diminuição significante do consumo nos animais do grupo que recebeu dieta hiperlipídica.

Segundo Flores et al..$^{26}$, os resultados indicam que o exercício físico interfere diretamente no hipotálamo e no controle do apetite. Observaram que o exercício físico aumenta a sensibilidade da leptina e da insulina no hipotálamo, suportando a hipótese de que o exercício físico levaria à situação de redução de apetite.

A diferença significante no teor de triglicerídeos entre os grupos indica que a dieta hiperlipídica isoladamente não aumentou a concentração de triglicerídeos plasmáticos. Achados semeIhantes foram observados por Morais et al. ${ }^{27}$, que atribuíram o fato ao aumento dos níveis de ácido linoléico e $\alpha$-linolênico na dieta, inferindo os efeitos hipotrigliceridêmicos, também sendo verificado por $\mathrm{Neves}^{28}$. Jong ${ }^{29}$ também observou redução nos níveis de triglicérides quando aumentou a concentração de $7 \%$ para 30\% de lipídios na dieta.

A diferença significante observada apenas entre os grupos NS e HE pode ter ocorrido devido às características díspares dos grupos. Nota-se ainda que o exercício físico não foi capaz de reduzir significantemente os níveis séricos de triglicerídeos tanto nos animais com dieta normoenergética (NS $\times$ NE) quanto nos animais com dieta hiperenergética (HS x NE).

O contrário foi observado em ratos treinados em corrida em esteira (65 a 70\% do máximo consumo de oxigênio) por Quiles et al. ${ }^{30}$, supondo que a intensidade do exercício utilizado (25\% a 
$65 \%$ do máximo consumo de oxigênio) tenha sido insuficiente para que o efeito hipotrigliceridêmico ocorresse.

O exercício mostrou-se eficaz em elevar significantemente os níveis de HDL-colesterol entre os grupos NS; NE e HS; NE $(p=0,0025)$, corrobo-rando os achados de Bernardes et al. ${ }^{31}$. Entretanto, isso foi verificado apenas nos animais com dieta normolipídica, contrariando o que cita $\mathrm{Katan}^{32}$, que sugere que a fração HDL-colesterol relaciona-se proporcionalmente ao conteúdo de gordura da dieta.

A partir dos resultados encontrados, conclui-se que as inalterações no peso corporal dos grupos estudados confirma que a dieta com $14 \%(p / p)$ de gordura não é suficiente para elevar o peso de ratos Wistar. A partir disso, os efeitos ergogênicos vinculados à dieta hiperlipídica não foram evidenciados, sendo que o tipo do exercício utilizado, a intensidade e o tempo podem ter sido também os fatores interferentes.

Apesar do maior consumo alimentar entre os animais normolipídicos, os níveis de triglicérides foram semelhantes estatisticamente aos valores dos animais hiperlipídicos. Essa semelhança pôde advir das concentrações mais elevadas de ácidos graxos poliinsaturados na dieta, evidenciando seus efeitos hipotrigliceridêmicos.

A interação dieta hiperlipídica - exercício, entretanto, não foi capaz de reduzir os níveis séricos de triglicerídeos, em comparação com os sedentários, sugerindo que a intensidade do exercício deve ser mais elevada para que ocorra a função de reduzir concentrações plasmáticas de gordura. Por outro lado, o exercício físico realizado foi significantemente válido no aumento do $\mathrm{HDL}$-colesterol entre os animais que receberam dieta normolipídica, confirmando a relevância da dieta equilibrada sobre esse parâmetro lipídico, associada ao exercício físico aeróbio.

\section{A GRADECIMENTO}

À Coordenação de Aperfeiçoamento de Pessoal de Nível Superior, pelo apoio financeiro.

\section{COLABORADORES}

L.D.P. FRANCO participou do desenvolvimento da pesquisa prática (cuidando dos animais) e da realização do levantamento dos dados da mesma. J.A.D.B. CAMPOS participou do desenvolvimento do artigo auxiliando no tratamento estatístico dos resultados. A. DEMONTE auxiliou na elaboração deste artigo supervisionando a redação e a análise dos dados.

\section{REFERÊ N CIAS}

1. Gaíva MH, Couto RC, Oyama LM, Couto GEC, Silveira VLF, Ribeiro EB, et al. Diets rich in polyunsaturated fatty acids: effect on hepatic metabolism in rats. Nutrition. 2003; 19(2):144-9.

2. Ellis J, Lake A, Hoover-Plow J. Monounsaturated canola oil reduces fat deposition in growing female rats fed a high or low fat diet. Nutr Res. 2002; 22(5):609-21.

3. Lombardo YB, Chicco AG. Effects of dietary polyunsaturated n-3 fatty acids on dyslipidemia and insulin resistance in rodents and humans: a review. J Nutr Biochem. 2006; 17(1):1-13.

4. Hill JO, Lin D, Yakubu F, Peters JC. Development of dietay obesity in rats: Influence of amount and composition of dietary fat. Int J Obes. 1992; 16(5): 321-33.

5. Ikemoto S, Takahashi M, Tsunoda N, Maruyama K, Itakura H, Ezaki O. High-fat diet-induced hyperglycemia and obesity in mice: differential effects of dietary oils. Metabolism. 1996; 45(12): 1539-46.

6. Jump DB. The biochemistry of $n-3$ polyunsaturated fatty acids. J Biol Chem. 2002; 277(11):8755-8.

7. Iritani N, Komiya M, Fukuda H, Sugimoto T. Lipogenic enzyme gene expression is quickly supressed in rats by a small amount of exogenous polyunsaturated fatty acids. J Nutr. 1998; 128(6): 967-72.

8. Yoshida H, Mawatani M, Ikeda I, Imaizumi K, Seto A, Tsuji H. Effect of dietary seal and fish oils on triacylglycerol metabolism in rats. J Nutr Sci Vitaminol (Tokyo). 1999; 45(4):411-21.

9. Powers SK, Howley ET. Fisiologia do exercício: teoria e aplicação ao condicionamento e ao desempenho. 3a. ed. São Paulo: Manole; 2000.

10. Curi R, Lagranha CJ, Hirabara SM, Folador A, Tchaikovski Jr. O, Fernandes LC, et al. Uma etapa limitante para a oxidação de ácidos graxos durante 
o exercício aeróbio: o ciclo de Krebs. Rev Bras Ciênc Mov. 2003; 11(2):87-94.

11. Coyle EE. Fat metabolism during exercise. Gatorade Sport Science Exchange. 1995; 8:1-6.

12. Newsholme EA. An introduction to the roles of the glucose-fatty acid cycle in sustained exercise. In: Maughan RJ, Shirreffs SM, editors. Biochemistry of exercise IX. Champaign: Human Kinetics Publishers; 1996.

13. Reeves PG, Nielsen FH, Fahey GC Jr. AIN-93 purified diets for laboratory rodents: final report of the American Institute of Nutrition ad hoc writing committee on the reformulation of the AIN-76A rodent diet. J Nutr. 1993; 123(11):1939-51.

14. Bligh EG, Dyer WJ. A rapid method of total lipid extraction and purification. Can J Biochem Physiol. 1959; 37(8):911-7.

15. Association of Official Analytical Chemists. Official methods of analysis. $16^{\text {th }}$ ed. Arlington: Association of Analytical Communities; 1995.

16. Lancha Junior AH, Recco MB, Abdalla DSP, Curi R. Effect of aspartate, asparagine and carnitine supplementation in the diet on metabolism of skeletal muscle during a moderate exercise. Physiol Behav. 1995; 57(2):367-71.

17. Pereira LO, Francischi RP, Lancha Jr. AH. Obesidade: hábitos nutricionais, sedentarismo e resistência à insulina. Arq Bras Endocrinol Metab. 2003; 47(2): 111-27.

18. Bell RR, Spencer MJ, Scherriff JL. Voluntary exercise and monounsaturated canola oil reduce fat gain in mice fed diets high in fat. J Nutr. 1997; 127(10): 566-73.

19. Pellizzon M, Buison A, Ordiz Jr F, Lardo SA, Jen KLC. Effects of dietary fatty acids and exercise on body-weight regulation and metabolism in rats. Obes Res. 2002; 10(9):947-55.

20. Cheng B, Karamizerak O, Noakes TD, Dennis SC, Lambert EV. Time course of changes in rat muscle enzymes involved in fat oxidation following exercise training and a high fat diet. Clin Sci. 1994; 87(Supplement):89-95.

21. Stich V, Glisezinski I, Berlan M, Bulow J, Galitzky J, Harant I, et al. Adipose tissue lipolysis is increased during a repeated bout of aerobic exercise. J Appl Physiol. 2000; 88(4):1277-83.

22. Romijn JA, Coyle EF, Sidossis L. Regulation of endogenous fat and carbohydrate metabolism in relation to exercise intensity and duration. Am J Physiol. 1993; 265(3Pt1):E380-E391.

23. Romijn JA, Coyle EF, Sidossis LS, Rosenblatt J, Wolfe RR. Substrate metabolism during different exercise intensities in endurance-trained women. J Appl Physiol. 2000; 88(5):1707-14.

24. Kretschmer BD, Schelling P, Beier N, Liebscher C, Treutel S, Krüger N, et al. Modulatory role of food, feeding regime and physical exercise on body weight and insulin resistance. Life Sci. 2005; 76(14): 1553-73.

25. Gaiva MH, Couto RC, Oyama LM, Couto GE, Silveira VL, Riberio EB, et al. Polyunsaturated fatty acid-rich diets: effect on adipose tissue metabolism in rats. Br J Nutr. 2001; 86(3):371-7.

26. Flores MBS, Fernandes MF, Ropelle E, Faria MC, Ueno $\mathrm{M}$, Velloso LA, et al. Exercise improves insulin and leptin sensitivity in hypothalamus of Wistar rats. Diabetes. 2006; 55(9):2254-61.

27. Morais CSN, Barcelos MFP, Sousa RV, Lima HM, Lima AL. Efeitos das fontes e níveis de lipídios nas dietas de ratos machos da linhagem Wistar (Rattus norvegicus) sobre frações lipídicas do sangue. Ciênc Agrotec. 2003; 5:1082-8.

28. Neves NM. Nutrição e doença cardiovascular. Rio de Janeiro: Guanabara Koogan; 1997.

29. Jong EV. Influência de dietas normo e hiperlipídicas sobre o perfil nutricional, parâmetros bioquímicos séricos e estruturais de ratos Wistar [tese]. Campinas: Universidade Estadual de Campinas; 1996.

30. Quiles JL, Huertas JR, Ochoa JJ, Battino M, Mataix J, Manàs M. Dietary fat (Virgin Olive Oil or Sunflower Oil) and physical training interactions on blood lipids in the rat. Nutrition. 2003; 19(4): 363-8.

31. Bernardes D, Manzoni MSJ, Sousa CP, Tenório N, Dâmaso AR. Efeitos da dieta hiperlipídica e do treinamento de natação sobre o metabolismo de recuperação ao exercício em ratos. Rev Bras Educ Fís Esporte. 2004; 18(2):191-200.

32. Katan MB. Effect of low-fat diets on plasma high-density lipoprotein concentrations. Am J Clin Nutr. 1998; 68(3Suppl):573S-76S.

Recebido em: 7/8/2007

Versão final reapresentada em: 22/4/2008 Aprovado em: 25/6/2008 